\title{
EL ACCESO AL AGUA POTABLE EN EL CHACO [Argentina] Y LOS PROGRESOS HACIA EL OBJETIVO DEL MILENIO. Una mirada a través de la elaboración de un índice de criticidad
}

\author{
ACCESS TO DRINKING WATER IN CHACO [Argentina] AND PROGRESS TOWARDS THE \\ MILLENNIUM GOAL. \\ A look through the elaboration of a criticality index.
}

\author{
Dra. Liliana Ramírez \\ Instituto y Departamento de Geografía \\ Facultad de Humanidades-UNNE \\ Prof. Titular ordinaria Técnicas en Geografía II. \\ Investigadora Independiente-CONICET \\ E-mail: Iramirez@hum.unne.edu.ar
}

Donde llega el agua hay riqueza y donde no, pobreza (proverbio).

La población mundial está creciendo a alrededor de 80 millones de personas al año, lo que implica una mayor demanda de agua dulce de aproximadamente

64 mil millones de metros cúbicos año.

La competencia por el agua existe en todos los niveles y se prevé que aumentará en casi todos los países http://www.unwater.org/statistics_use.html (27oct2013)

\section{Resumen}

La disponibilidad de agua dulce está considerada como uno de los factores determinantes de la salud humana, en los últimos decenios, el crecimiento de la población, la industrialización y el incremento de la utilización de agua para la producción ha ocasionado un fuerte aumento en la demanda de este recurso. Si bien en el mundo hay suficiente agua dulce, la dificultad radica en la desigual distribución en el tiempo y en el espacio ya que en muchos países se está empleando con niveles insostenibles. Ésta -y otras cuestiones que preocupan- han llevado a la formulación de los Objetivos de Desarrollo del Milenio (ODM) aprobados en la Declaración del año 2000 de Naciones Unidas. Según estos objetivos, el $92 \%$ de la población en América Latina y el Caribe debiera tener acceso al agua potable en el año 2015, ésta es la Meta 7C que forma parte del "Objetivo 7 (ODM7). Argentina, al igual que todos los países signatarios, reconoce a estos objetivos y a las metas que los componen como las aspiraciones e ideales de desarrollo de la sociedad en su conjunto.

Recientemente se han publicado en la página oficial del Instituto Nacional de Estadística y Censos de la Argentina datos que fueron relevados el 27 de octubre de 2010, ocasión en que se llevo a cabo el $10^{\circ}$ Censo Nacional de Población. Hogares y Viviendas. La asequibilidad y el procesamiento de los datos on-line permite recuperar información relevante para el conocimiento de las características de las condiciones de vida de la población de las localidades, municipios y departamentos de todas las provincias argentinas.

En este aporte queremos acercar un análisis referido a la procedencia de agua para beber y cocinar en los municipios de la provincia del Chaco, con el propósito de apreciar cuán cerca [o no] nos encontramos de alcanzar el objetivo planteado por Naciones Unidas. A la vez se intentará establecer la relación entre las necesidades actuales y los proyectos existentes para ampliar el acceso al agua por red en nuestra provincia.

\section{Palabras Claves}

Chaco - Agua Potable - Accesibilidad - Criticidad - Objetivos de Desarrollo del Milenio

\section{Abstract}

The availability of drinking water is considered a determinant of the human health, in the last decades, the population increase, the industrialization and the increment of the water use for the production,

Publicado en formato digital: Dra. Liliana Ramírez. EL ACCESO AL AGUA POTABLE EN EL CHACO [Argentina] Y LOS PROGRESOS HACIA EL OBJETIVO DEL MILENIO. Una mirada a través de la elaboración de un índice de criticidad. Revista Geográfica Digital. IGUNNE. Facultad de Humanidades. UNNE. Año 10. No 20. Julio Diciembre 2013. ISSN 1668-5180 Resistencia, Chaco. En: http://hum.unne.edu.ar/revistas/geoweb/default.htm 
caused soaring demand expansion is it. In the world drinking water is sufficient; the difficulty is in the unequal distribution in the time and in the space, as a lot of countries use the water with unsustainable.

These and other issues determined that the United Nations made the Millennium Development Goals, it is approved in Declaration of year 2000. According to these objectives, in 2015, the $92 \%$ of population in Latin America and Caribbean have got access drinking water, this is goal 7C of the 'Objective 7'. Argentina like all signatories countries, admit this is goal and objectives as the aspirations and ideals of the development of society as a whole.

Recently published in the official website of National Institute of Statistics and Censuses of Argentina data were relieved in October of 2010, in occasion of the tenth national census of population, home and housing. The accessibility and processing of data on-line allows retrieving relevant information for knowledge of the characteristics of the living conditions of the population of the towns, municipalities and departments of all Argentinian provinces.

In this contribution, we want to bring an analysis referred to the source of water for drinking and cooking in the municipalities of the province of Chaco, with the purpose to determine how close (or not) we are to achieve the goal set by United Nations, while we try to provide the relationship between the current needs and the existing projects to expand access to drinking water in our province.

\section{Key Words}

Chaco - Drinking Water - Accessibility - Criticality - Millennium Development Goals (MDGs)

6 MARS 2012 | GINEBRA/NUEVA YORK - El mundo ha cumplido con la meta de los Objetivos de Desarrollo del Milenio (ODM) de reducir a la mitad la proporción de personas sin acceso al agua potable mucho antes de la fecha límite de 2015, según un informe publicado hoy por UNICEF y la Organización Mundial de la Salud (OMS).

El informe, Progress on Drinking Water and Sanitation 2012 (Progreso sobre el agua potable y saneamiento 2012), publicado por el Programa Conjunto para el Monitoreo del Abastecimiento de Agua $y$ Saneamiento, de UNICEF y la OMS, dice que a finales de 2010 un 89\% de la población mundial, o 6.100 millones de personas, utilizaban fuentes mejoradas de agua potable. Se trata de un 1\% más que la cifra que figuraba en la meta de los ODM, un 88\%. El informe estima que, en 2015 , el $92 \%$ de la población mundial tendrá acceso al agua potable (OMS, 2012).

\section{Introducción}

El acceso universal al agua potable está considerada como una meta del Objetivo del Milenio $n^{\circ} 7$ que señala que es preciso "garantizar la sostenibilidad del medio ambiente", esta declaración de Naciones Unidas aprobada por todos los países signatarios, nos exige monitorear este indicador de manera recurrente, con el propósito de apreciar los avances en el sentido señalado por el objetivo. Al respecto, en un comunicado de prensa del 12 de marzo de 2012 la OMS señalaba:

Este año, en el reporte 2013 de los ODM presentado por Naciones Unidas, se señala que a pesar de los avances sin precedentes que se han logrado, el 83\% de la población sin acceso a una fuente mejorada de agua potable (636 millones de personas) vive en áreas rurales. Más aún, sigue inquietando la calidad y la seguridad de muchas de las fuentes mejoradas de agua potable. En consecuencia, la cantidad de personas sin acceso al agua potable segura podría ser dos o tres veces superior a las estimaciones oficiales (ONU, ODM, 2013).

Monitorear permanentemente estas estimaciones es de alta relevancia, sobre todo si consideramos que la disponibilidad de agua, de saneamiento y de higiene tienen consecuencias importantes sobre la salud y la enfermedad de la población, además de estos tres aspectos es importante considerar la cantidad de agua domiciliaria de la que se dispone. No hay estimaciones oficiales respecto de la cantidad de agua diaria que se requiere para satisfacer las necesidades básicas y también para promover una buena salud. En 2003 en un estudio de la OMS se consideraba que 7,5 litros per cápita por día atenderían las necesidades de la mayoría de las personas en casi todas las condiciones, señalaba además que la calidad de esta agua debe tener un nivel tolerable de riesgo (OMS; 2003);

Publicado en formato digital: Dra. Liliana Ramírez. EL ACCESO AL AGUA POTABLE EN EL CHACO [Argentina] Y LOS PROGRESOS HACIA EL OBJETIVO DEL MILENIO. Una mirada a través de la elaboración de un índice de criticidad. Revista Geográfica Digital. IGUNNE. Facultad de Humanidades. UNNE. Año 10. No 20. Julio Diciembre 2013. ISSN 1668-5180 Resistencia, Chaco. En: http://hum.unne.edu.ar/revistas/geoweb/default.htm 
Sin embargo otro estudio más reciente de la ONU sugiere que cada persona necesita de 20 a 50 litros de agua al día para asegurar sus necesidades básicas para beber, cocinar y limpiar (ONU, 2013).

Retomando las consideraciones sobre el ODM a cumplir, sería una satisfacción extraordinaria si el cumplimiento del objetivo global llegara a todas las latitudes del planeta, pero las distintas realidades regionales y locales distan de aquel alcance. Es importante reconocer los esfuerzos realizados para acercarnos a la meta que se persigue, sin embargo, al analizar los datos recientemente publicados, advertimos que la provincia se encuentra aún por debajo del ODM y que las diferencias a nivel local siguen siendo difíciles de superar. Para mostrar esta realidad, en la presente contribución, ilustraremos la situación provincial y las particularidades que presenta el Chaco a nivel municipal en un intento por dejar al descubierto las desigualdades territoriales y, en consecuencia, aquellas áreas y sociedades que por su situación merecen un tratamiento diferente para alcanzar la equidad en el acceso al agua potable.

\section{Fuentes, materiales y métodos}

Para el monitoreo de los ODM, la OMS propone indicadores que sean fácilmente actualizables y comparables, en el caso del ODM7 [meta C], se trata de la "proporción de la población con acceso a fuentes mejoradas de abastecimiento de agua potable".

Es por ello que en virtud de la disponibilidad de los datos recientemente publicados por el INDEC, hemos realizado un análisis comparativo de la situación en la que se encontraban los municipios en los años 2001 y 2010.

De lo expresado se deduce que la fuente de datos estadísticos es el Censo 2001 y el Censo 2010, en ambos casos se accedió a la Base de Datos Redatami, disponible en el sitio oficial del Instituto Nacional de Estadística y Censos (www.indec.gov.ar).

Para el análisis, en primer lugar, se consideraron las proporciones simples de cada categoría de la variable "procedencia del agua en vivienda" para el año 2001 y "procedencia del agua para beber y cocinar" para el año 2010, deteniéndonos exclusivamente en las características del acceso por red pública. De este modo se pueden distinguir cuáles son los municipios que ya han superado el ODM, los que están cerca de cumplirlo y los que requieren de un mayor esfuerzo para alcanzar a la meta en 2015.

Complementariamente a las proporciones simples, hemos ensayado un indicador o índice de criticidad de la "procedencia de agua para beber y cocinar" considerando todas las categorías que incluye esta variable. Se trata de una construcción estadística que tiene como origen los datos de porcentajes de todas las categorías de la variable "procedencia de agua para beber y cocinar" en cada uno de los municipios de la provinciaii. El valor porcentual de cada categoría fue estandarizó y el acceso al agua por red pública fue la única categoría que se consideró como beneficio para la población, mientras que las otras categorías tuvieron una consideración opuesta. En otras palabras, aquella categoría cuyo incremento implica una mejor situación de la población [red pública] resta valor a la criticidad, mientras que las categorías cuyo incremento implica peor situación de la población [perforaciones, pozos, cisternas, agua de lluvia, río, canal, arroyo] suman valor al índice final de criticidad. La notación aritmética del Índice de Criticidad de Acceso al Agua potable (ICAA) sería:

$I C A A=\sum(S t R P+S t P B M t+S t P B M n+S t P z+S t T C+S t L I C R A A)$

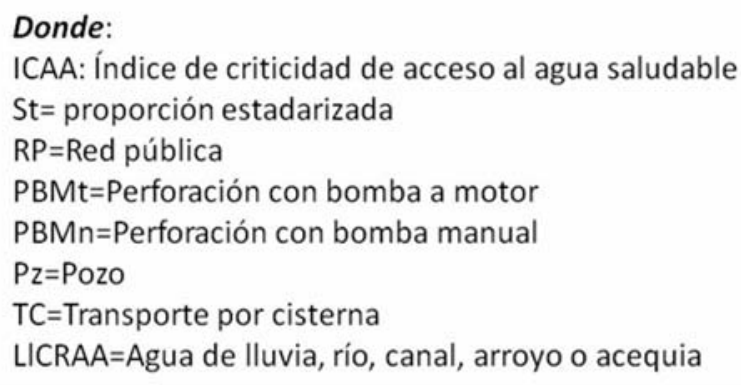

Figura 1: Notación del Índice de criticidad de acceso al agua saludable

Publicado en formato digital: Dra. Liliana Ramírez. EL ACCESO AL AGUA POTABLE EN EL CHACO [Argentina] Y LOS PROGRESOS HACIA EL OBJETIVO DEL MILENIO. Una mirada a través de la elaboración de un índice de criticidad. Revista Geográfica Digital. IGUNNE. Facultad de Humanidades. UNNE. Año 10. No 20. Julio Diciembre 2013. ISSN 1668-5180 Resistencia, Chaco. En: http://hum.unne.edu.ar/revistas/geoweb/default.htm 
El mayor valor que podría haber alcanzado el ICAA es 6, esto implicaría que las seis variables incluidas en él alcanzaran el valor máximo posible que es 1, es decir la situación más desfavorable. Sin embargo, tras el cálculo los valores oscilaron entre 0 y 4,04, más adelante profundizaremos.

Finalmente, la perspectiva geográfica y el principio [rector] de localización que nuestra ciencia sostiene, nos exige a que hagamos visibles las desigualdades territoriales a través de representaciones cartográficas, ésta es la forma más genuina de apreciar cuál es el patrón de distribución en el acceso al agua y poder observar, a través de un mapa, las áreas que merecen mayores esfuerzos de gestión por parte de los gobiernos, por esta razón se observará la implantación del índice a través de la cartografía correspondiente.

\section{Resultados}

\section{a. ¿Cuánto avanzamos? ¿Cuánto nos falta?}

Si se comparan los datos censales de 1991, 2001 y 2010 el porcentaje de viviendas con acceso a agua segura de red pública ha presentado mejoras sustanciales a nivel nacional tanto en términos absolutos como relativos $(68,3 \%, 78,4 \%$ y $86,2 \%$ respectivamente). Esta tendencia también se manifiesta en la Provincia del Chaco que revela un aumento del 50,63\% al 76,54\% de viviendas con acceso a agua de red pública entre los últimos dos censos (ver gráfico 1). No obstante, aunque en menor proporción, algunas categorías también muestran un incremento, por ejemplo, las perforaciones con bomba manual y el transporte de agua por cisterna, en el primero de estos dos casos se trata de una decisión individual que los pobladores asumen para alcanzar el suministro necesario, mientras que en el segundo caso se trata de una estrategia desarrollada por cooperativas, por gobiernos locales o por el gobierno provincial que acercan, de forma regular, el agua a los hogares. Paralelamente disminuyeron los hogares que se abastecen de agua de pozo -con y sin bomba-, de agua de lluvia, canal, río o arroyo y de perforaciones con bomba a motor, destacándose que en el primer y segundo caso la disminución fue significativa.

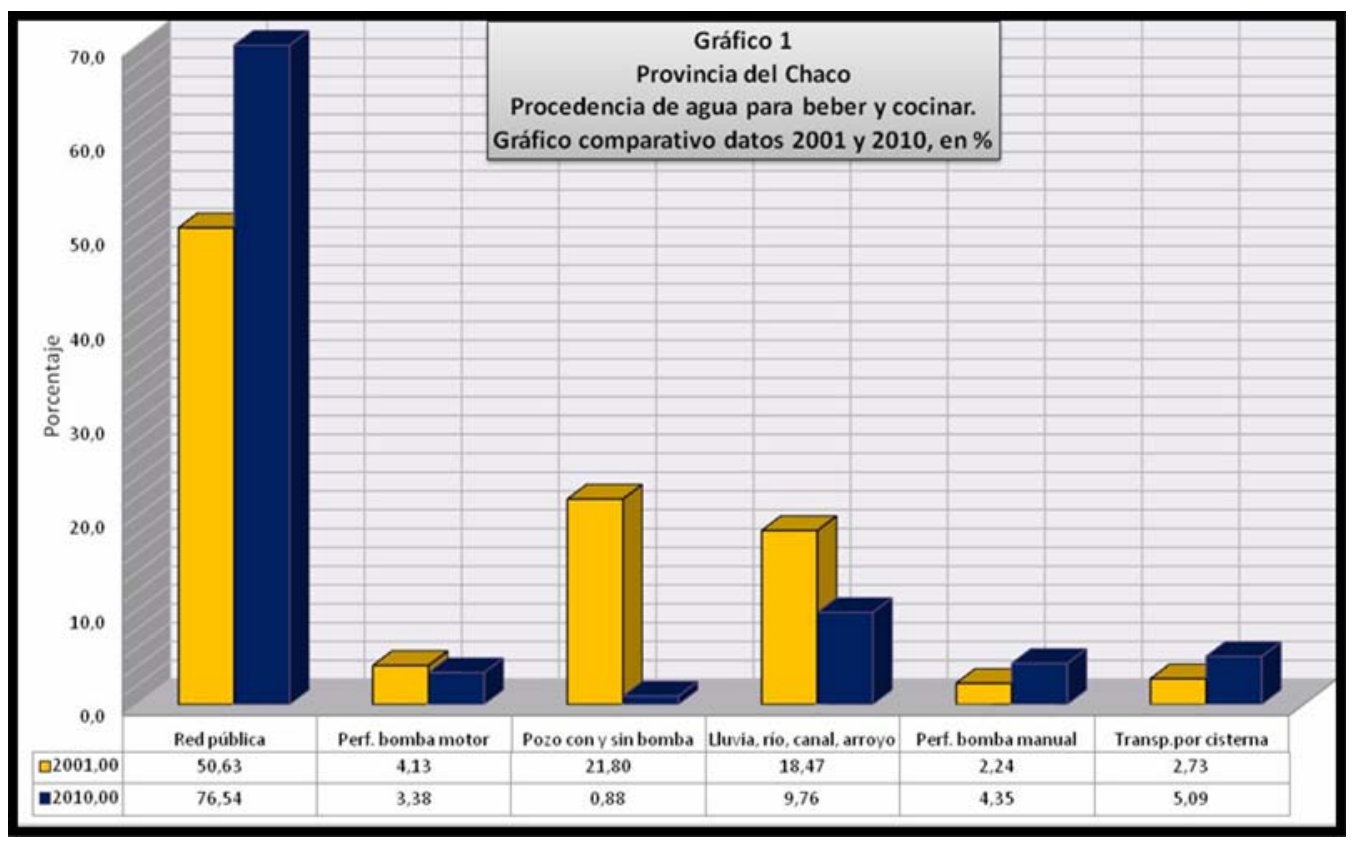


Sin duda alguna la tendencia hacia a mejores situaciones de abastecimiento de agua potable es

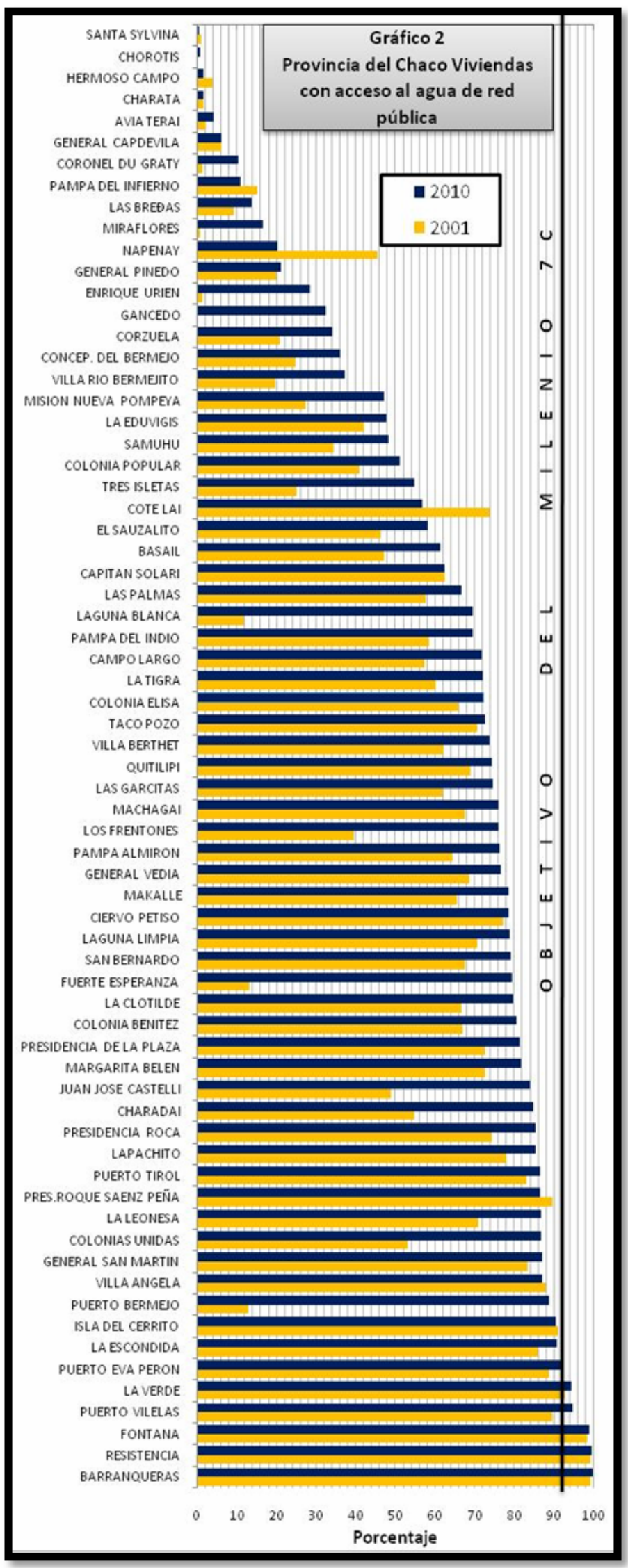
evidente, sin embargo también es preciso reconocer las diferencias que se visualizan hacia adentro de un territorio provincial, ya que muchas veces, los datos a escalas jurisdiccionales no dejan percibir los problemas a escala local o municipal y mucho menos a una microescala barrial. Esta desigualdad es muy evidente si apreciamos los datos que se encuentran en el gráfico 2, en color claro el porcentaje de viviendas con acceso a agua por red pública en 2001 y en color oscuro en 2010.

Visualizando el gráfico 2 el primer comentario que podemos realizar es que 6 municipios ya han alcanzado el ODM7C [igual o mayor al 92\%], a saber: Barranqueras, Resistencia, Fontana, Puerto Vilelas, La Verde y Puerto Eva Perón. Respecto de los que aún no llegaron al valor que se espera para el 2015, el gráfico arroja la visualización de la gran diversidad de situaciones que presenta el Chaco, algunos municipios seguramente alcanzaran a cumplir el objetivo mientras que otros, por más esfuerzos que se realicen difícilmente logren conseguirlo.

En otro orden de análisis hay que destacar que 58 municipios mejoraron el acceso al agua de red. Dentro de este grupo hay 5 municipios que podrían considerarse sin modificaciones ya que su incremento es inferior al 1\%, ellos son: Charata, Resistencia, Barranqueras, Fontana y Chorotis. En el otro extremo con un crecimiento superior al $20 \%$ de viviendas con acceso en 2010 respecto de 2001, se aprecia un conjunto de 12 municipios: Misión Nueva Pompeya, Enrique Urien, Tres Isletas, Charadai, Gancedo, Colonias Unidas, Juan José Castelli, Los Frentones, Laguna Blanca, Fuerte Esperanza, Puerto Bermejo, los últimos tres con más del $50 \%$ de incremento. Entre ambos extremos más de cuarenta municipios mejoraron su cobertura entre un 1 y un $18 \%$.

Sin lugar a dudas, los datos analizados demuestran un importante crecimiento, detrás del cual hay un gran esfuerzo que implica una mejora muy significativa no sólo en pos del cumplimiento de un objetivo global que persiguen todos los países, sino en pos de mejorar la calidad

Publicado en formato digital: Dra. Liliana Ramírez. EL ACCESO AL AGUA POTABLE EN EL CHACO [Argentina] Y LOS PROGRESOS HACIA EL OBJETIVO DEL MILENIO. Una mirada a través de la elaboración de un índice de criticidad. Revista Geográfica Digital. IGUNNE. Facultad de Humanidades. UNNE. Año 10. No 20. Julio Diciembre 2013. ISSN 1668-5180 Resistencia, Chaco. En: http://hum.unne.edu.ar/revistas/geoweb/default.htm 
de vida de la población y responder a las características que actualmente tienen los municipios saludables. Sin embargo nuestro compromiso con la realidad nos exige mostrar las desigualdades, las cuestiones pendientes, ya que estas [más que los logros], son las que deben considerarse para seguir avanzando.

En efecto, los municipios que merecen profundizar todo tipo de programas, proyectos y acciones concretas para mejorar la situación son: General Pinedo, Napenay, Miraflores, Las Breñas, Pampa Del Infierno, Coronel Du Graty, General Capdevila, Avia Terai, Charata, Hermoso Campo, Chorotis y Santa Sylvina, en todos ellos el acceso al agua por red no alcanza el 25\%. Esto implica un abastecimiento a través de cisternas, perforaciones, almacenamiento de agua de lluvia, entre otras formas de acceso. Esta difícil circunstancia se profundiza todavía más si consideramos que gran parte del sector occidental del Chaco tiene una elevada concentración de arsénico y la acumulación del agua suele realizarse en recipientes no adecuados, todo ello propicia situaciones poco favorables para garantizar la salud de la población.

b. ¿Cómo podemos visualizar las diferencias territoriales del índice de criticidad? ¿Dónde hay que profundizar los esfuerzos?

Tal como se describió en el apartado metodológico, para analizar el acceso al agua a nivel territorial se consideraron todas las posibles provisiones y se logró el índice de criticidad para cada municipio. Si bien el análisis presentado en el apartado anterior llevó implícita la dimensión espacial ya que se mencionaron los municipios con ciertas particularidades, en este ítem queremos hacer explícita la visualización de la distribución geográfica.

El mapa 1 muestra el indicador de criticidad a través de coropletas, utilizando la clasificación basada en cuartiles (17 municipios en cada categoría). El primer cuartil es el que engloba a las jurisdicciones en condiciones más propicias, mientras que el cuarto cuartil contiene a los menos beneficiados. Es muy evidente la desfavorable situación que caracteriza al sudoeste chaqueño.

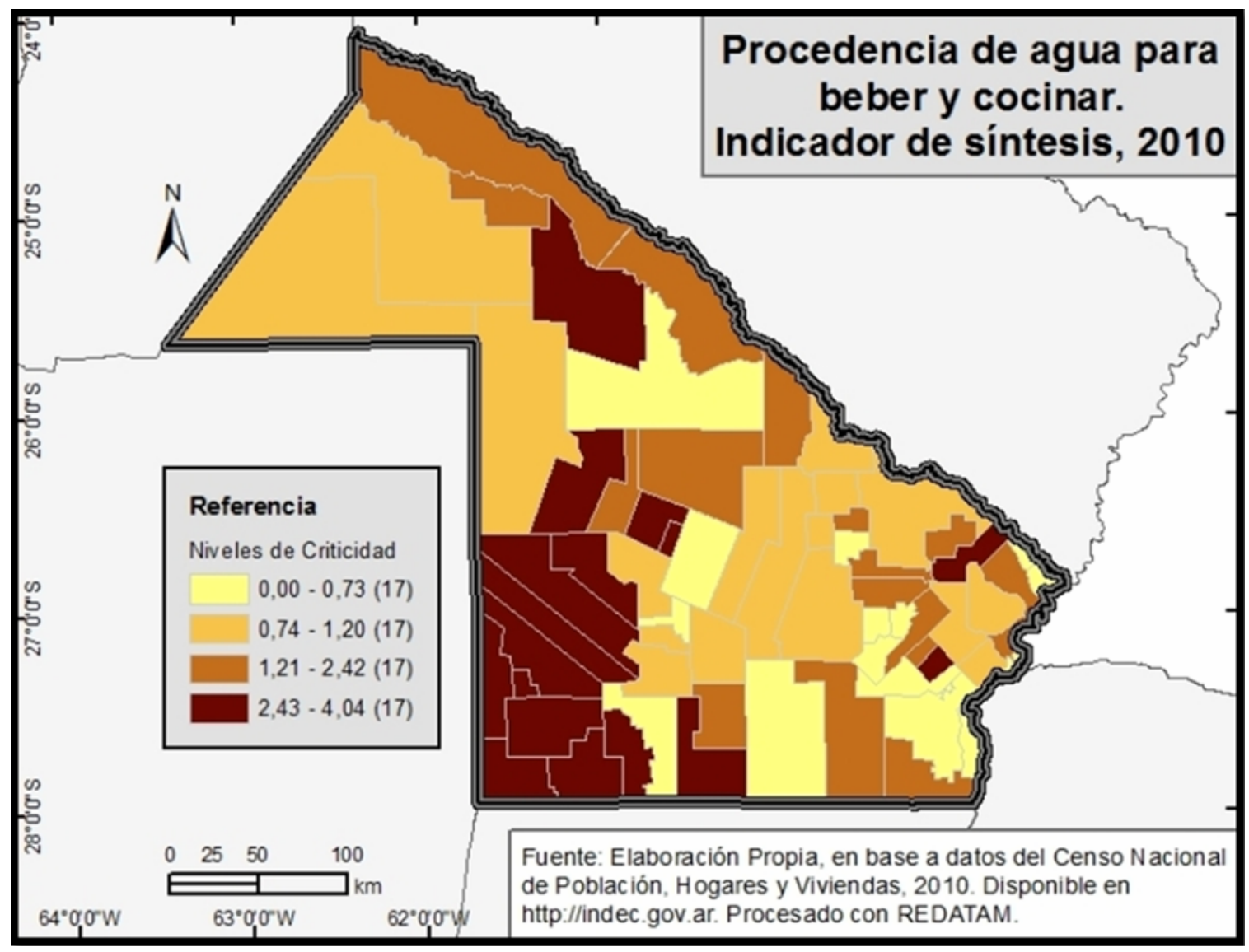

Mapa 1 
Para describir lo que sucede territorialmente, como comentamos anteriormente, dividimos a los 68 municipios en cuartiles. Del primer cuartil, con las situaciones más beneficiosas (o menos desfavorables), forman parte los municipios de: La Clotilde, Lapachito, Puerto Tirol, Juan José Castelli, Charadai, Presidencia Roque Sáenz Peña, Colonias Unidas, Villa Ángela, Puerto Bermejo, La Escondida, Isla del Cerrito, Puerto Eva Perón, La Verde, Puerto Vilelas, Fontana, Resistencia y Puerto Barranqueras; en estos casos el acceso al agua a través de red pública, se encuentra entre valores que van desde un $79,8 \%$ en La Clotilde a un $99,7 \%$ en Barranqueras, en total reúnen el $56,2 \%$ de la población chaqueña. Hay que señalar que en este grupo el segundo tipo de provisión en importancia es el agua de pozo y en tercer lugar el abastecimiento de agua de lluvia, río, arroyo o canal.

El segundo cuartil que agrupa el $19 \%$ de la población provincial se encuentra compuesto por los municipios de Laguna Limpia, Taco Pozo, La Tigra, Margarita Belén, Colonia Benítez, Campo Largo, Quitilipi, Presidencia De La Plaza, Machagai, Las Garcitas, Villa Berthet, Los Frentones, La Leonesa, Fuerte Esperanza, General San Martin, San Bernardo, Presidencia Roca; en esta unidades la provisión de agua por red se encuentra entre un $71,9 \%$ y un $87,2 \%$; se advierte que comienza a ser más significativo el abastecimiento de agua de pozo alcanzando el máximo valor en La Tigra con un $21,9 \%$ y en tercer lugar, la obtención de agua de perforación con bomba a motor.

El tercer cuartil que aglutina el 10,3\% de la población se encuentra formado por los municipios de Basail, Concepción del Bermejo, Villa Rio Bermejito, Las Palmas, Misión Nueva Pompeya, Laguna Blanca, Samuhú, Capitán Solari, Cote Lai, Tres Isletas, El Sauzalito, Pampa Almirón, Colonia Elisa, Ciervo Petiso, Pampa Del Indio, Makallé y General Vedia. Este conjunto de municipios es más diverso en cuanto a la provisión de agua de red pública, ya que oscila entre un valor mínimo de $36,2 \%$ y un registro máximo de $78,7 \%$, no obstante sus semejanzas tienen que ver con la importancia relativa que comienzan a evidenciar las restantes o categorías o tipos de abastecimiento de agua, en este sentido aparece el primer municipio en el que no predomina el agua por red, se trata de Concepción del Bermejo en donde el $39,1 \%$ de los hogares se sustentan con agua de pozo y el $36,2 \%$ con agua de red.

Finalmente, el grupo menos favorecido, el cuarto cuartil, está conformado por Charata, Santa Sylvina,

Chorotis, Hermoso Campo, General Capdevila, Avia Terai, Coronel Du Graty, Pampa Del Infierno, Las Breñas, Miraflores, General Pinedo, La Eduvigis, Napenay, Enrique Urien, Gancedo, Corzuela, Colonia Popular. Si nos remitimos al mapa que se acompaña es el grupo que presenta una mayor continuidad territorial, y su localización, casi exclusiva en el sudoeste chaqueño, deja en clara evidencia el principal problema de la región. En este conjunto sólo en dos municipios, que precisamente no están en el sudoeste (La Eduvigis y Colonia Popular), es mayoría la provisión de agua por red; en todos los otros casos, la principal forma de abastecimiento se reparte entre; agua de

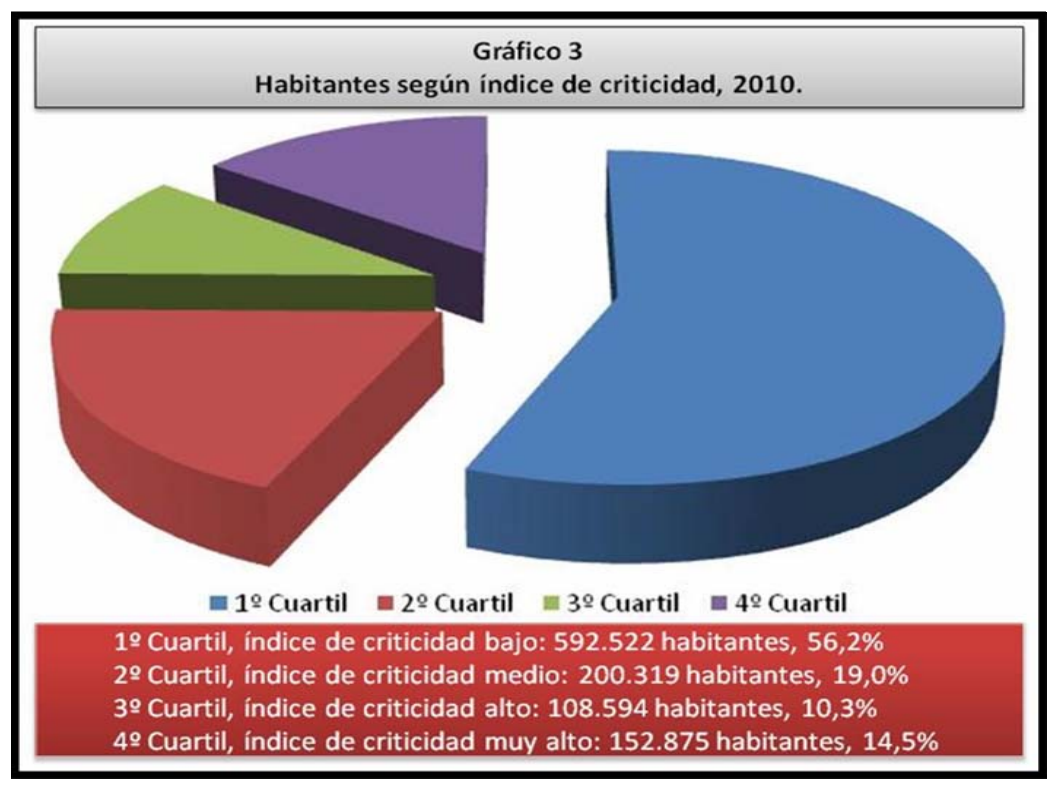
pozo (en 7 municipios), agua de lluvia, río, canal o arroyo (en 5 municipios) y agua transportada a través de cisternas (en 3 municipios). Estas últimas categorías predominantes en el sudoeste se combinan de diferentes maneras y abastecen siempre a más de la mitad de la población. Este grupo, el más comprometido desde de la perspectiva de la provisión de agua potable reúne el $14,5 \%$ de la población provincial, unas 153.290 personas.

El gráfico 3 sintetiza la descripción realizada en términos de población incluida en cada uno de los cuatro grupos, podemos apreciar la mayoría ya descripta en el primero de ellos con mejores condiciones de acceso, sin embargo no hay

Publicado en formato digital: Dra. Liliana Ramírez. EL ACCESO AL AGUA POTABLE EN EL CHACO [Argentina] Y LOS PROGRESOS HACIA EL OBJETIVO DEL MILENIO. Una mirada a través de la elaboración de un índice de criticidad. Revista Geográfica Digital. IGUNNE. Facultad de Humanidades. UNNE. Año 10. № 20. Julio Diciembre 2013. ISSN 1668-5180 Resistencia, Chaco. En: http://hum.unne.edu.ar/revistas/geoweb/default.htm 
que descuidar los dos últimos conjuntos que contienen un valor cercano al 25\% de la población chaqueña, éstos son los que merecerían mayores esfuerzos gubernamentales para superar la dificultad en el acceso al agua saludable.

Si bien el índice de criticidad se calculó para cada uno de los municipios y se trata de un valor global de la jurisdicción, tenemos que tener en cuenta que, según el censo 2010, la población urbana promedio de la provincia superó el $87 \%$, por lo tanto la situación más desfavorable, además de lo ya señalado, estaría concentrada en la localidades que forman parte de la red urbana provincial. En el mapa 2 se ha pretendido mostrar la territorialización del índice de criticidad con especial énfasis en los asentamientos urbanos, se destacan como ciudades con prioridad a ser atendidas, las localidades del sudoeste, Avia Terai en el centro y Colonia Popular en el sector oriental de la Provincia.

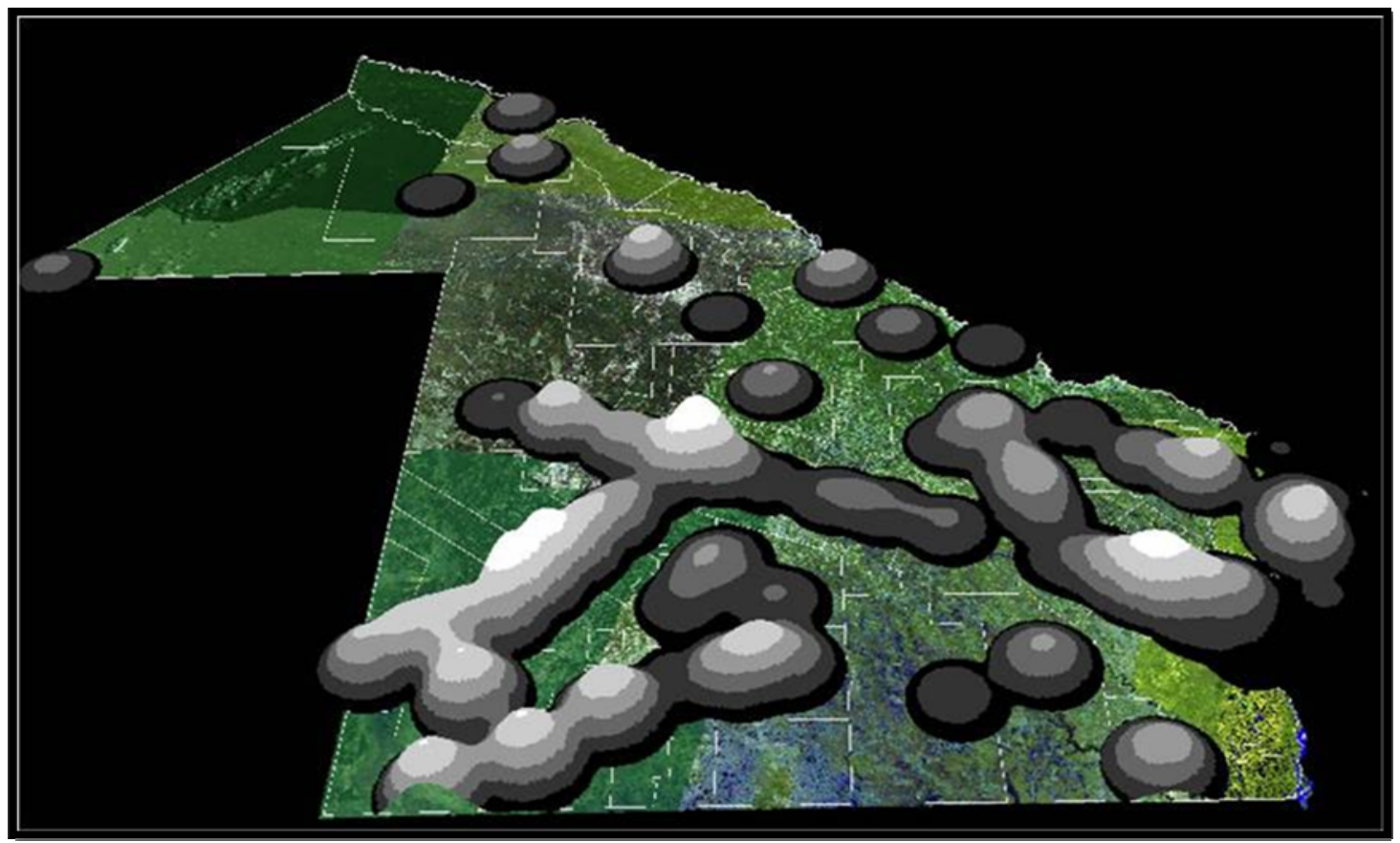

\section{Mapa 2}

\section{c. Localización de los acueductos actuales y proyectados}

La solución a la falta o escasez de agua ha sido, desde tiempos pretéritos, la construcción de acueductos. Esta palabra o término como la conocemos en la actualizad, tiene su origen en el antiguo imperio romano [Aquaeductus (Aqvaedvctvs)], era la forma habitual con la que se designaba a un conducto que transportara agua, no importando si era a través de un canal, un puente, una galería o de una cañería (BUITRAGO LEÓN, 2004). Durante la Edad Media los árabes con sus canales de riego y de abastecimiento a poblaciones persiguieron el bienestar agrícola y la higiene. De la misma manera en la América Latina, obras de igual importancia y majestuosidad en el área de abastecimiento de agua y sistemas de riego fueron construidas por las comunidades Indígenas, que habitaban entre otros países, México (Azteca y Maya), Colombia (Tierra dentro, Sinu, etc.), Perú y Bolivia (Inca). Con la llegada de los españoles muchas de estas construcciones y aspectos técnicoculturales fueron destruidos, y solo hasta hace algunos anos los arqueólogos e investigadores han empezado a recuperar esta parte importante de nuestra Historia y acervo cultural (BUITRAGO LEÓN, 2004).

La provincia del Chaco tiene una larga historia de proyectos de acueductos, sin embargo, sólo algunos se han concretado permitiendo incrementar el acceso al agua saludable y con el ello mejorar la salud y aumentar la calidad de vida de la población. En el mapa que sigue (mapa 3) hemos representado la red de acueductos de la provincia; como se puede observar, los que actualmente están en funcionamiento, representados con la categoría "existentes", benefician a una porción muy reducida del territorio, su proyección hasta Santa Sylvina y Villa Berthet se encuentra "en ejecución" y "a ejecutarse" respectivamente. El más destacado, y esperado por toda la población, es el "segundo acueducto", cuya extensión de 510 kilómetros beneficiará, -particularmente- al sudoeste de la 
provincia, se encuentra actualmente "licitado". El escenario se completa con otros proyectos de menor extensión, no por ello menos importantes, cuyas características se pueden ver en la Tabla 1.

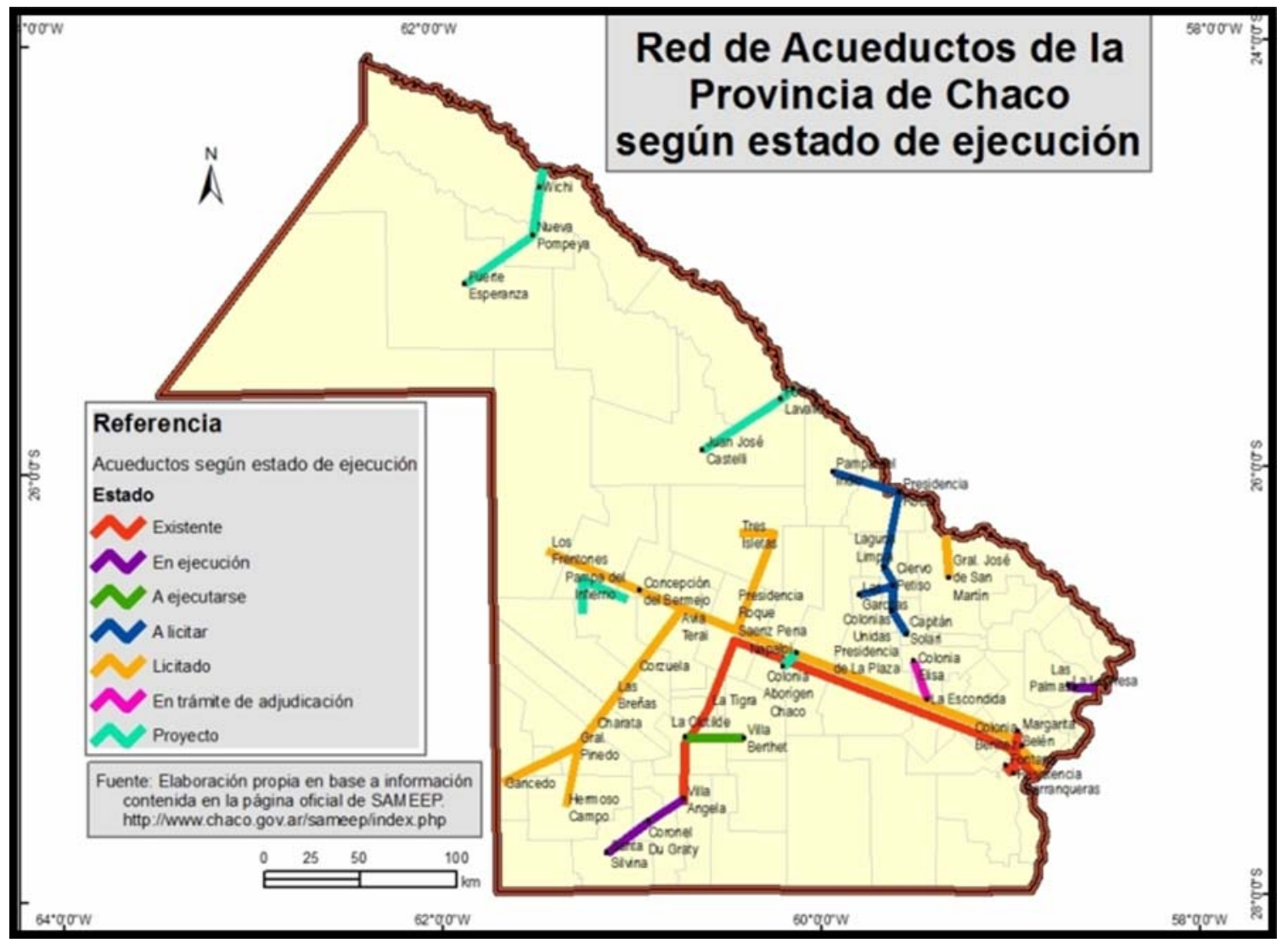

Mapa 3

\begin{tabular}{|l|c|c|}
\hline \multicolumn{1}{c|}{ DENOMINACIÓN } & ESTADO & LONGITUD \\
\hline Primer acueducto & Existente & $270 \mathrm{~km}$ \\
\hline Resistencia-Margarita Belén-Colonia Benítez & Existente & $28 \mathrm{~km}$ \\
\hline Resistencia-Fontana & Existente & $5 \mathrm{~km}$ \\
\hline La Leonesa-Las Palmas & En ejecución & $10 \mathrm{~km}$ \\
\hline Villa Ángela-Coronel Du Graty-Santa Sylvina & En ejecución & $57 \mathrm{~km}$ \\
\hline San Bernardo-Villa Berthet & A ejecutarse & $28 \mathrm{~km}$ \\
\hline Segundo acueducto & Licitado & $510 \mathrm{~km}$ \\
\hline Rio Bermejo-General San Martin & Licitado & $24 \mathrm{~km}$ \\
\hline La Escondida-Colonia Elisa & En trámite de adjudicación & $26 \mathrm{~km}$ \\
\hline $\begin{array}{l}\text { Presidencia Roca-Pampa del Indio-Laguna .Limpia- } \\
\text { Las Garcitas-Ciervo Petiso-Colonias Unidas Capitán } \\
\text { Solari }\end{array}$ & A Licitar & $151 \mathrm{~km}$ \\
\hline Pintado-Wichi-Nueva Pompeya-Fuerte Esperanza & & \\
\hline Puerto Lavalle-Fortín Lavalle-Juan José Castelli & Proyecto & $84 \mathrm{~km}$ \\
\hline Napalpí-Colonia Aborigen & Proyecto & $63 \mathrm{~km}$ \\
\hline $\begin{array}{l}\text { Loro Blanco-Pampa del Infierno-Concepción del } \\
\text { Bermejo }\end{array}$ & Proyecto & $14 \mathrm{~km}$ \\
\hline
\end{tabular}

Tabla 1: Red de acueductos en la Provincia del Chaco

Publicado en formato digital: Dra. Liliana Ramírez. EL ACCESO AL AGUA POTABLE EN EL CHACO [Argentina] Y LOS PROGRESOS HACIA EL OBJETIVO DEL MILENIO. Una mirada a través de la elaboración de un índice de criticidad. Revista Geográfica Digital. IGUNNE. Facultad de Humanidades. UNNE. Año 10. № 20. Julio Diciembre 2013. ISSN 1668-5180 Resistencia, Chaco. En: http://hum.unne.edu.ar/revistas/geoweb/default.htm 
Revista Geográfica Digital. IGUNNE. Facultad de Humanidades. UNNE. Año 10. № 20.

Julio - Diciembre 2013. ISSN 1668-5180 Resistencia, Chaco

La expansión de la red de acueductos que lleven agua potable, saludable y segura a los hogares tiene externalidades positivas que han sido ampliamente difundidas en diversas publicaciones. Los efectos directos más evidentes se demuestran en la salud de la población, especialmente en aquellos segmentos más vulnerables sin la posibilidad de acceder al agua por otros medios que no sea la red pública. Se ha demostrado de manera fehaciente la disminución de la morbilidad y de la mortalidad infantil [y general] de origen hídrico e infecto-contagiosas, cuando la población accede al agua seguraiii. En el caso del Chaco por ejemplo, la morbilidad por causas infecto-contagiosas ha disminuido a nivel provincial, sin embargo en un reciente estudio se ha demostrado que las que persisten se concentran hacia el noroeste, ya que el centro medio que las representa se ha desplazado, entre 2000 y 2007, 40,3 kilómetros hacia ese punto cardinal (Cfr. Ramírez, 2012). Reforzando esta idea, en 2012, la Directora General de la OMS, Dra. Margaret Chan, señaló que "proporcionar un acceso sostenible a fuentes mejoradas de agua potable es una de las cosas más importantes que podemos hacer para reducir las enfermedades" (OMS, 2012). Éstas, entre otras razones, son las que han determinado que tanto el acceso al agua como al saneamiento se han incorporado a los ODM.

En definitiva, un elevado número de personas, asientan la esperanza de mejorar su estado sanitario y calidad de vida en la construcción de los acueductos, mientras tanto se depende de la distribución a través de las cooperativas, de las bondades de la naturaleza cuando llueve y se puede almacenar o de las reiteradas perforaciones hasta encontrar alguna que no supere el nivel de arsénico permitido por la OMS.

\section{Conclusiones}

Para concluir queremos resaltar que en la Argentina, en 2010, el 83\% de la población tenía acceso a agua por red pública, esto representaba unos 32,8 millones de personas. A nivel provincial, sólo tres jurisdicciones se encuentran con un valor inferior al del Chaco (recordemos 76,5\%), Santiago del Estero (75,8\%), Provincia de Buenos Aires (75,1\%) y Misiones (71,9\%), mientras que once jurisdicciones ya han superado la meta que propone el Objetivo del Milenio (recordemos, 92\%). Es importante reconocer los esfuerzos realizados para acercarnos a la meta que se persigue, sin embargo, para menguar las diferencias a nivel local, que siguen siendo difíciles de superar, se requerirá de estrategias, recursos y políticas focalizadas que minimicen las disparidades intraprovinciales y, fundamentalmente, concretar la construcción de los acueductos que garanticen el acceso a nivel local y las cinco condiciones que deber tener el acceso al agua: suficiente, saludable, aceptable, físicamente accesible y asequible.

Para monitorear los avances de las naciones signatarias, la ONU cuenta con cuatro programas específicos: (i) El Programa mundial de evaluación de los recursos hídricos; (ii) El Programa conjunto OMS/UNICEF de monitoreo del abastecimiento de agua y del saneamiento; (iii) El Programa de ONUAgua para el desarrollo de la capacidad en el marco del Decenio; y (iv) El Programa de ONU-Agua sobre fomento y comunicación en el marco del Decenio. Estos programas nos informan permanentemente lo que sucede a nivel global y ello nos posibilita tener un horizonte hacia dónde observar los acontecimientos mundiales.

A nivel local, como hemos visto alrededor de 150.000 personas en el Chaco son las que se encuentran más afectadas por la falta de acceso al agua, esto influye directamente en el consumo de agua y alimentos asépticos, en la salud y en la higiene personal, a lo que también hay que añadir la disponibilidad de este recurso para las actividades productivas. Los datos, cuadros, gráficos y representaciones presentados en los apartados anteriores echan luces respecto de la situación a escala provincial, pero más aún sobre las realidades locales que es donde más se siente la necesidad de agua saludable. Concretar los proyectos de acueductos que proporcionen agua potable a la población es una obligación de los estados miembros de las Organización de las Naciones Unidas que el 28 de julio de 2010, a través de la Resolución 64/292, reconoció explícitamente el derecho humano al agua y al saneamiento, reafirmando que un agua potable, limpia y el saneamiento son necesarios para la realización de todos los derechos humanos.

Publicado en formato digital: Dra. Liliana Ramírez. EL ACCESO AL AGUA POTABLE EN EL CHACO [Argentina] Y LOS PROGRESOS HACIA EL OBJETIVO DEL MILENIO. Una mirada a través de la elaboración de un índice de criticidad. Revista Geográfica Digital. IGUNNE. Facultad de Humanidades. UNNE. Año 10. № 20. Julio Diciembre 2013. ISSN 1668-5180 Resistencia, Chaco. En: http://hum.unne.edu.ar/revistas/geoweb/default.htm 
Revista Geográfica Digital. IGUNNE. Facultad de Humanidades. UNNE. Año 10. № 20. Julio - Diciembre 2013. ISSN 1668-5180 Resistencia, Chaco

\section{Referencias citadas}

BUITRAGO LEÓN, I. (2004). Aportes para la historia de los acueductos. Disponible en: http://www.agualatinoamerica.com/docs/pdf/050604\%20Acueductos.pdf

CEPAL, 2010. Avance regional hacia el cumplimiento de los Objetivos de Desarrollo del Milenio.

INDEC, 2010. Censo Nacional de Población, Hogares y Vivienda. Datos en línea. www.indec.gov.ar.

LENTINI, E. y BRENER, F., 2012. Agua y Saneamiento: un Objetivo de Desarrollo de Milenio. Los avances en Argentina. Voces en el Fénix $N^{\circ} 20$. Facultad de Ciencias Económicas. Universidad Nacional de Buenos Aires.

OMS, 2003. La cantidad de agua domiciliaria, el nivel del servicio y la salud. Guy Howard, Water Engineering and Development Centre, Universidad de Loughborough, RU, y Jamie Bartram, Organización Mundial de la Salud, Ginebra, Suiza.

http://www.who.int/water_sanitation_health/diseases/wsh0302/es/index.html

OMS, 2012. Se cumple la meta sobre agua potable del Objetivo de Desarrollo del Milenio La meta de saneamiento sigue estando muy retrasada. Disponible en:

http://www.who.int/mediacentre/news/releases/2012/drinking_water_20120306/es/

OMS, 2013. Objetivos de Desarrollo del Milenio. Informe 2013.

http://www.un.org/es/millenniumgoals/pdf/mdg-report-2013-spanish.pdf

ONU, 2010. Decenio Internacional para la acción "El agua fuente de vida 2005-2015". Disponible en: http://www.un.org/spanish/waterforlifedecade/human_right_to_water.shtml

ONU, 2013. Lo programas de ONU Agua. Disponible en:

http://www.unwater.org/discover_es.html\#action

RAMíREZ, M. L. 2012. Morbilidad en la Provincia del Chaco (2000-2007). Aproximación a la distribución, dispersión y difusión de las principales causas de enfermedades de la población. En: Foschiatti, A.M. (2012) "Escenarios vulnerables de Nordeste Argentino". UNNE, ANPCYT, CONICET.

\section{Sitios Web:}

http://www.who.int/globalchange/ecosystems/water/es/

http://www.raco.cat/index.php/treballsscgeografia/article/viewFile/184519/237797

http://www.unwater.org/index.html

http://www.unwater.org/statistics_san.html\#sthash.wKLx9Y6V.dpuf

\footnotetext{
${ }^{i}$ Redatam es el acrónimo de REcuperación de DATos para Áreas pequeñas por Microcomputador. Redatam+SP (abreviado $\boldsymbol{R}+\boldsymbol{S P}$ ), la última versión en homenaje a su creador Serge Poulard y originalmente lanzado como Redatam+G4 $(R+G 4)$, es la cuarta generación del software, que puede ser usada en español, inglés o portugués, con Microsoft Windows 9x, NT4, 2000 y superiores, en cualquier microcomputador compatible con IBM (winR+ v.1.2 es la versión previa, que opera también con Windows 3.1).

ii Las categorías de la variables "procedencia del agua para beber y cocinar" son: *Red pública, *Perforación con bomba a motor, *Perforación con bomba manual, *Pozo, *Transporte por cisterna, *Agua de lluvia, río, canal, arroyo o acequia.

iii De acuerdo con estimaciones realizadas a nivel mundial, progresos en el suministro de agua puede reducir la morbilidad por diarrea en un $21 \%$, el saneamiento mejorado en un $37,5 \%$, el lavado de mano hasta un $35 \%$ y mejoras adicionales en la calidad de agua potable, como la desinfección en el punto de consumo, pueden reducir los episodios diarrericos hasta un 45\% (Lentini y Brenner, 2012).
} 